\title{
MONITORING LACTOFERRIN IRON LEVELS BY FLUORESCENCE RESONANCE ENERGY TRANSFER: A COMBINED CHEMICAL AND COMPUTATIONAL STUDY
}

Fernando Carmona, Víctor Muñoz-Robles, Rafael Cuesta, Natividad Gálvez, Mercè Capdevila, Jean-Didier Maréchal, José M. Dominguez-Vera

Abstract.Three forms of lactoferrin (Lf) that differed in their levels of iron loading (Lf, LfFe, and $\mathrm{LfFe}_{2}$ ) were simultaneously labeled with the fluorophores AF350 and AF430. All three resulting fluorescent lactoferrins exhibited fluorescence resonance energy transfer (FRET), but they all presented different FRET patterns. Whereas only partial FRET was observed for Lf and LfFe, practically complete FRET was seen for the holo form $\left(\mathrm{LfFe}_{2}\right)$. For each form of metal-loaded lactoferrin, the AF350-AF430 distance varied depending on the protein conformation, which in turn depended on the level of iron loading. Thus, the FRET patterns of these lactoferrins were found to correlate with their iron loading levels. In order to gain greater insight into the number of fluorophores and the different FRET patterns observed (i.e., their iron levels), a computational analysis was performed. The results highlighted a number of lysines that have the greatest influence on the FRET profile. Moreover, despite the lack of an X-ray structure for any LfFe species, our study also showed that this species presents modified subdomain organization of the N-lobe, which narrows its iron-binding site. Complete domain rearrangement occurs during the $\mathrm{LfFe}$ to $\mathrm{LfFe}_{2}$ transition. Finally, as an example of the possible applications of the results of this study, we made use of the FRET fingerprints of these fluorescent lactoferrins to monitor the interaction of lactoferrin with a healthy bacterium, namely Bifidobacterium breve. This latter study demonstrated that lactoferrin supplies iron to this bacterium, and suggested that this process occurs with no protein internalization.

Keywords: Lactoferrin, Iron metabolism, Protein-ligand docking, FRET, Structural analysis 


\section{Introduction}

Lactoferrin is a glycoprotein $(80 \mathrm{kDa})$ of the transferrin family with a high affinity for iron(III) [1, 2]. Lactoferrin possesses various biological functions, including antibacterial, antiviral, and antiparasitic activities [3]. High lactoferrin levels are found in colostrum and milk, and this protein is also present in most mucosal secretions, including uterine fluid, vaginal, and nasal secretions, as well as in tears $[4,5]$.

The extraordinary affinity of lactoferrin for iron undoubtedly determines part of its functionality. Indeed, lactoferrin is considered to form part of the innate immune system due to its effects on pathogen growth. Iron is essential for life and is a key nutrient for pathogenic microorganisms, which require this metal to survive and replicate. Life can to some extent be considered a battle for iron, so hosts must deprive undesirable guests of iron in order to combat the infections they cause. As a result, iron uptake by lactoferrin prevents the development of pathogenic microorganisms which cannot then access this metal. This is why blood lactoferrin levels increase markedly during infection. Indeed, lactoferrin concentrations increase in all biological fluids during most inflammatory reactions and viral infections, with the highest levels being detected at the nidus of the inflammation [6].

The clearest evidence that the immune function of lactoferrin is related to its iron affinity is the fact that whereas apolactoferrin (lactoferrin without iron) inhibits the growth of a large number of species of bacteria (such as Escherichia coli, some Pseudomonas, Yersinias, Listeria, Streptococcus, and Staphylococcus), hololactoferrin (iron-saturated lactoferrin) exhibits significantly lower inhibitory activities towards such bacteria [7-9]. The iron loading level of lactoferrin therefore partially determines its functionality.

It should be noted that lactoferrin also has some effects on the immune system that have nothing to do with iron affinity. Thus, lactoferrin exerts antiviral effects by binding directly to DNA (or RNA) and by preventing the entry of viruses into cells, thereby stopping infections at an early stage [10-13].

Lactoferrin can also serve as an iron donor, supporting the growth of certain beneficial bacteria $[14,15]$. This occurs in breast milk, where lactoferrin, 
Lactobacilli, and Bifidobacteria coexist in an environment that is optimized for proliferation.

X-ray diffraction studies, which are only available for the apo and holo forms of lactoferrin, have shown that it consists of one polypeptide chain containing 703 amino acids, which forms two homologous globular domains named the $\mathrm{N}$ - and $\mathrm{C}$ lobes. The N-lobe corresponds to amino acid residues 1-333 and the C-lobe to residues 345-692, and the ends of these domains are connected by a short ahelix (Fig. 1). These two homologous lobes are further subdivided into two similarly sized domains known as domain 1 and domain 2 (domains $\mathrm{N} 1$ and N2 in the N-lobe and domains C1 and C2 in the C-lobe), and the two iron-binding sites are located within the interdomain clefts of each lobe. In human lactoferrin, when two iron atoms are incorporated into the apoprotein, the C-lobe conformation does not change significantly whereas the N-lobe closes like a hinge to grip the metal. This results in different tertiary structures: iron-free apolactoferrin is characterized by an open conformation of the N-lobe and a closed conformation of the C-lobe, whereas both lobes are closed in the iron-rich hololactoferrin, as shown by X-ray.

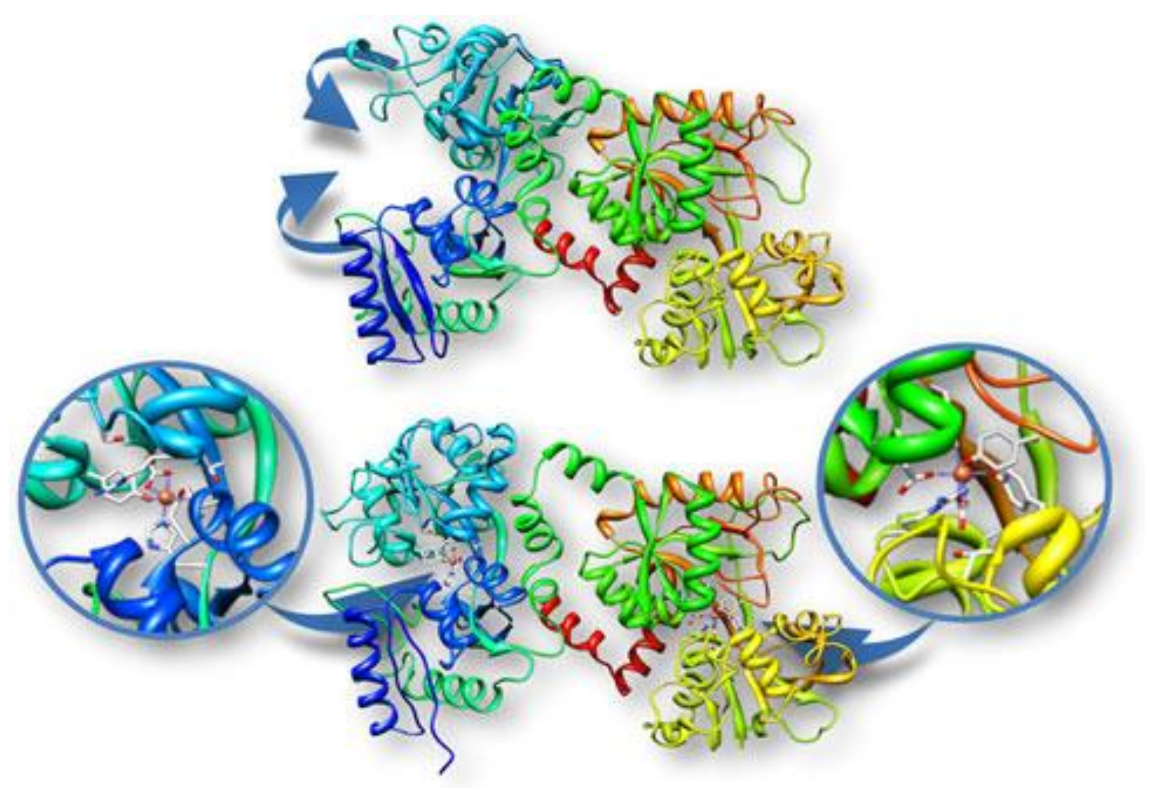

Fig. 1 X-ray structures of Lf and LfFe2. Arrows indicate the conformational movement of the $N$-lobe when coordinating iron. The iron-binding sites are magnified to highlight the chemical environment of the iron at each site 
The chemical environments of the two iron(III) coordination sites are similar and consist of two phenolate oxygens from two tyrosine residues, one imidazole from a histidine residue, one carboxylate from an aspartate residue, and a synergistic adjacent bicarbonate or carbonate, which is stabilized by hydrogen bonding to the peptide chains that surround the active site. Despite this similarity, the two coordination sites of iron(III) can be distinguished kinetically and spectroscopically [18]. The three known forms of lactoferrin, namely apolactoferrin (Lf), the monoferric form (LfFe), and hololactoferrin $\left(\mathrm{LfFe}_{2}\right)$ differ in their iron loading levels, which determine the functionality of the protein.

The lysine residues on the external surface of lactoferrin can be used to covalently couple molecules [19-22] or clusters [23], permitting new functionality to be added to the protein. This option has been exploited for different applications, including increasing cell internalization levels, oral administration, rheology modulation, and for monitoring toxic non-transferrin bound iron. We use this functionalization to determine the iron loading level of the protein. The idea of incorporating a fluorescent moiety into lactoferrin is of considerable and widespread interest, especially in the field of biomedicine, as adequate functionalization could lead to fluorescence resonance energy transfer (FRET), an effect/process that is commonly used in sensors in biological research [24-26].

In the work described in the present paper, a pair of Alexa Fluor ${ }^{\circledR}$ (AF) fluorophores, AF350 and AF430, were chemically bound to Lf, LfFe, and $\mathrm{LfFe}_{2}$. As the emission band of the AF350 donor overlaps with the excitation band of the AF430 acceptor, simultaneous labeling of these lactoferrin species with both fluorophores (AF350 and AF430) offers the opportunity to achieve FRET, which is strongly dependent on the distance between the AF350 donor and the AF430 acceptor. We show in this work that the FRET yield, which is the ratio of the emission intensities for the acceptor and donor, differs for Lf, LfFe, and $\mathrm{LfFe}_{2}$ because the conformational changes that the protein undergoes during iron incorporation lead to variations in the donor-acceptor distance. As a result, the FRET pattern can be used as an indicator of the tertiary structure of lactoferrin, thus providing information on its iron load. Finally, we made use of this correlation between the FRET pattern and iron loading level to determine the iron 
loading state of lactoferrin during its interaction with probiotic bacteria, a process which ends with the transfer of iron from the lactoferrin to the bacteria.

Moreover, we performed a computational study to rationalize this dependency of the FRET pattern on the iron loading level. The reason that this study is interesting is that the evolution of the FRET pattern with the tertiary structure of the protein can be used to probe how, upon the uptake of the first iron(III) ion, the other lobe of the protein is prompted to take up the second iron(III) ion. No definitive answer is available to this question at present.

\section{Materials and methods}

Monoferric lactoferrin (LfFe) was purchased from Fonterra (Auckland, New Zealand; ref. no: 81665295/100). The apo and holo forms were prepared from this monoferric lactoferrin.

Preparation of apolactoferrin (Lf)

Lyophilized human milk LfFe $(20 \mathrm{mg} / \mathrm{mL})$ was totally desaturated of iron following successive dialysis steps against $0.1 \mathrm{M}$ citric acid/citrate buffer (pH 3.50) using a high retention seamless cellulose tubing membrane with a molecular weight cutoff (MWCO) of 12,000 Da. The citric acid/citrate buffer was changed several times until no characteristic iron lactoferrin absorbance at $464 \mathrm{~nm}\left(\varepsilon^{464}=\right.$ $2,500 \mathrm{M}^{-1}$ ) was observed. The resulting colorless pale apolactoferrin solution was then dialyzed against phosphate buffer $\mathrm{pH} 6.80$ and subsequently chromatographed (Sephadex G-25, GE Healthcare, Little Chalfont, UK). The concentration of apolactoferrin was measured by UV/vis spectroscopy based on the absorbance at $280 \mathrm{~nm}\left(\varepsilon^{280}=92,300 \mathrm{M}^{-1}\right)$.

Preparation of diferric lactoferrin (LfFe2)

Based on a previously published procedure [27], $20 \mathrm{mg}$ of lyophilized human milk LFe were dissolved in $1 \mathrm{~mL}$ of TRIS-Cl buffer $\mathrm{pH} 6.80$ containing $5 \mathrm{mM}$ sodium bicarbonate. This solution was then titrated with $\mathrm{Fe}$ citrate at $37{ }^{\circ} \mathrm{C}$ under continuous stirring until the appearance of a characteristic dark reddish solution. Fe citrate was freshly prepared every day following a previously reported 
protocol [28].

Iron contents per lactoferrin were calculated directly by UV/vis spectroscopy from the relationship between the absorbance at $464 \mathrm{~nm}\left(\varepsilon^{464}=2,500 \mathrm{M}^{-1}\right)$ and that $280 \mathrm{~nm}\left(\varepsilon^{280}=92,300 \mathrm{M}^{-1}\right)$. The iron/protein values obtained were 0 (Lf), 1.09 (LfFe), and $1.97\left(\mathrm{LfFe}_{2}\right)$. These values were obtained from triplicate preparations, with less than $8 \%$ variation from the mean observed. The same lactoferrins were used for all subsequent experiments. This means that the iron content of every lactoferrin remained constant throughout the experiment.

\section{Preparation of fluorescent lactoferrins}

Lf, LfFe, or $\mathrm{LfFe}_{2}(2 \mathrm{~mL}, 20 \mathrm{mg} / \mathrm{mL})$ was incubated in a buffered PBS solution at pH 8 with an excess of (a) AF350, (b) AF430, and finally (c) a mixture of the AF350 and AF430 fluorophore succinimidyl ester derivatives. The two former assays allowed the molar ratio required for the third reaction to be determined. Specifically, an AF350:AF430:protein molar ratio of 30:15:1 was used to prepare the samples. The labeling reactions were performed at room temperature under continuous stirring for $24 \mathrm{~h}$. The resulting solutions were then exhaustively dialyzed at room temperature for 3 days against several changes of Milli-Q water (Millipore, Billerica, MA, USA) using a high retention seamless cellulose tubing membrane with a MWCO of $12,000 \mathrm{Da}$, and size exclusion chromatography (Sephadex G-25) was then performed to remove the unbound fluorophores. Protein-containing fractions were isolated. For apolactoferrin samples labeled with both fluorophores, the concentration of AF430 was directly calculated from the absorbance values of the UV-visible spectra at $430 \mathrm{~nm}\left(\varepsilon^{430}=16,000 \mathrm{M}^{-1}\right)$ because the absorbances of apolactoferrin and AF350 are negligible at this wavelength. The concentration of AF350 was obtained from the absorbance at $350\left(\varepsilon^{350}=19,000 \mathrm{M}^{-1}\right)$ by subtracting the absorbance of AF430 at this wavelength. The concentration of lactoferrin was obtained directly from the absorbance at $280 \mathrm{~nm}\left(\varepsilon^{280}=92,300 \mathrm{M}^{-1}\right)$ because the absorbances of both fluorophores at this wavelength can be considered negligible. A similar procedure was performed to determine the number of fluorophores for $\mathrm{LfFe}$ and $\mathrm{LfFe}_{2}$, with the UV-visible spectrum of the starting lactoferrin subtracted from each labeled lactoferrin spectrum beforehand. The average number of fluorophores per protein was determined after performing the reaction in triplicate. Values were 
very consistent (with less than $5 \%$ variation from the mean) and were rounded to the nearest integer (Table 1). FRET yields from the three samples were also calculated, and standard deviations from the average were less than $3 \%$.

Table 1 Number of fluorophores per lactoferrin (rounded to the nearest integer)

\begin{tabular}{lllll}
\hline Protein & Only AF350 & Only AF430 & $\begin{array}{l}\text { Combined use of both } \\
\text { fluorophores }\end{array}$ \\
\cline { 3 - 5 } & & & AF350 & AF430 \\
\hline Lf & $6(6.16)$ & $6(6.11)$ & $3(3.06)$ & $3(3.02)$ \\
LfFe & $5(5.09)$ & $5(5.09)$ & $3(3.10)$ & $2(2.12)$ \\
LfFe $_{2}$ & $5(5.09)$ & $5(4.98)$ & $3(3.07)$ & $2(1.97)$ \\
\hline
\end{tabular}

The average value calculated after performing the reaction in triplicate is shown in parentheses

All UV-visible spectra were recorded with a Thermo Scientific (Waltham, MA, USA) Spectronic Unicam UV300 spectrophotometer, and all fluorescence spectra were recorded with a Varian (Palo Alto, CA, USA) Cary Eclipse fluorescence spectrophotometer.

Incubation of AF-labeled lactoferrins with Bifidobacterium breve

Human milk B. breve (10 mg/mL; Biosearch S.A., Granada, Spain; CECT7263) was incubated with LfFe2-AFs ( $\left.4.86 \times 10^{-6} \mathrm{M}\right)$ under anaerobic conditions at $37^{\circ} \mathrm{C}$ in Hank's solution for 1, 2, 3, and $6 \mathrm{~h}$. Bacterial suspensions were then centrifuged at 4,000 rpm and the supernatant solutions were analyzed using a Varian Cary Eclipse fluorescence spectrophotometer. No fluorescence was observed from the bacterial pellets. The iron concentrations of the supernatant solutions were measured by ICP, with values of $>10^{-9} \quad M$ obtained. Likewise, the protein concentrations in the supernatant solutions remained constant (in the range 4-5 x $10^{-6} \mathrm{M}$, as measured by UV/vis spectroscopy based on the absorbance at $280 \mathrm{~nm}$; $\left.\varepsilon^{280}=92,300 \mathrm{M}^{-1}\right)$.

\section{Docking study}

Protein-ligand dockings were performed using the program GOLD 5.1. A harmonic restraint of $5.5 \mathrm{~kJ} / \mathrm{mol}$ was added between the $\mathrm{Ne}$ of the corresponding lysine 
(Lys301 or Lys296) and the C of the carboxylate group of the fluorophore. In the simulations where Lys301 or Lys296 were flexible, the Dunbrack rotamer library was used. The cavity was defined as a $20-\AA ̊$ sphere around Tyr82.

Structural statistics

The two scripts used in this work were written in Python and ported to the UCSF Chimera environment. Both can be obtained for free from the authors.

\section{Supplementary Material (ESM)}

Solvent Accessible Surface of the lysine residues in Lf

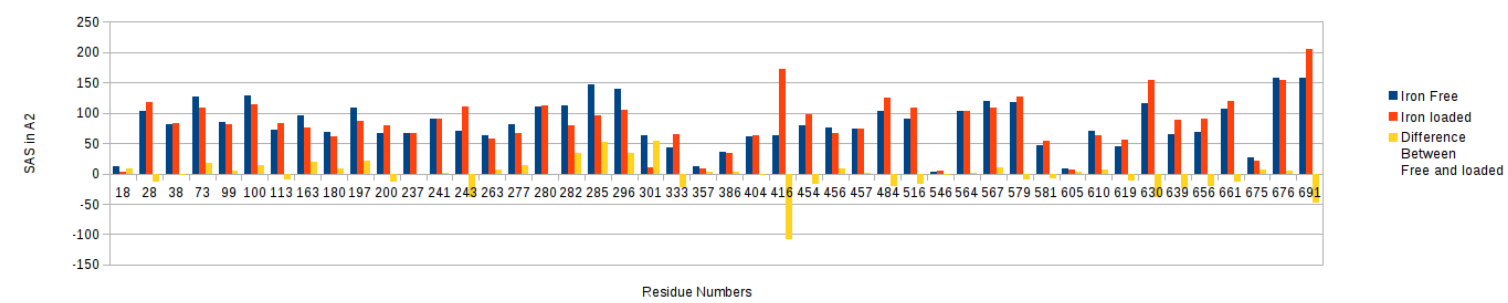

Figure SI1. Histogram of SAS values of the 46 Lys residues in Lf. Blue bars corresponds to values obtained for the Lf structure (pdb 1CB6), red bars to LfFe2 structure ( $p d b 1 N 76)$ and yellow bars to the relative difference between iron free and iron loaded conformations.

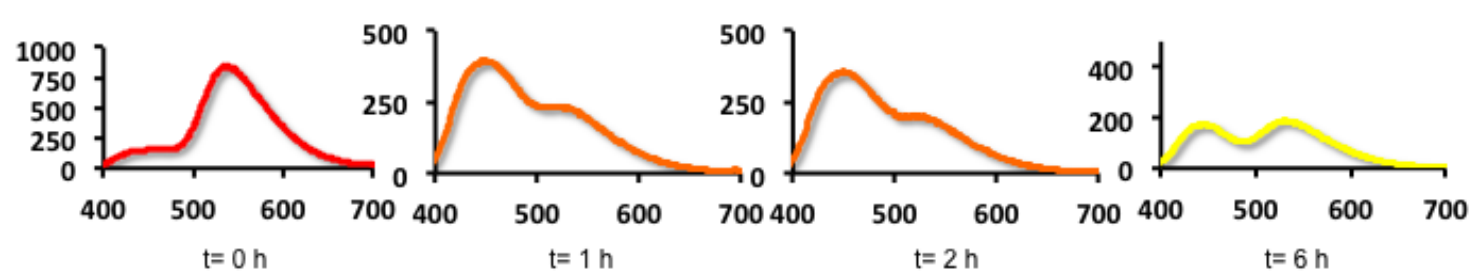

Figure SI2. Monitorization of FRET Fluorescence emission spectra for LfFe2-AFs before (left) and after (1, 2 and 6 h) incubation with Bifidobacterium breve. 


\section{Results}

Following a known procedure, apolactoferrin (Lf) and hololactoferrin (LfFe2) were prepared from the commercial monoferric form (LfFe). These three forms of lactoferrin with different iron loadings were treated with an excess of each AF fluorophore (or a mixture of both AF fluorophores) for $24 \mathrm{~h}$ and then exhaustively dialyzed until no fluorescence was detected in the dialysis reservoir. After isolating the protein-containing fractions, measurement of the UV-vis spectra allowed the final concentrations of each lactoferrin sample and the AF430 and AF350 fluorophores to be calculated. These results yielded the average number of fluorophores per lactoferrin, as shown in Table 1.

Our observations of the reactions of Lf, LfFe, and $\mathrm{LfFe}_{2}$ with AF350 and AF430 allowed us to establish that the number of reaction sites does not depend on the type of fluorophore used, and that they should be reacted with the proteins to determine the stoichiometric ratio (see "Materials and methods"). The fluorescence spectra of the solely AF350- and AF430-labeled lactoferrins showed emission bands centered at 445 and $540 \mathrm{~nm}$ upon excitation at 327 and $425 \mathrm{~nm}$, respectively. As expected, the fluorescence properties of AF350 and AF430 were not significantly modified after covalent coupling to lactoferrin.

AF350 and AF430 were chosen for use in this work due to the fact that the emission band of AF350 overlaps with the excitation band of AF430. Therefore, the simultaneous labeling of lactoferrin with both fluorophores (AF350 and AF430) is a good option for achieving FRET. The lactoferrins (Lf, LfFe, and $\mathrm{LFe}_{2}$ ) were finally incubated with an excess of a mixture of AF350 and AF430, using the same procedure as employed for the single AFs. The number of AF430 and AF350 moieties in each labeled sample was calculated from the absorbances at 430 and $350 \mathrm{~nm}$ in the UV-visible spectra (Fig. 2; see "Materials and methods").

As shown in Table 1, incubation of the lactoferrins ( $\mathrm{Lf}, \mathrm{LfFe}$, and $\mathrm{LFe}_{2}$ ) with an excess of a mixture of AF350 and AF430 yielded similar labeling results. The number of AF350 and AF430 molecules when the lactoferrins were labeled with a single fluorophore was six for Lf and five for both LfFe and $\mathrm{LfFe}_{2}$. Interestingly, the number of AF350 and AF430 molecules in the combined experiments was also found to be also six (3 AF350 + 3 AF430) for Lf-AFs, five (3 AF350 + 2 AF430) for 
LfFe-AFs, and five (3 AF350 + 2 AF430) for LfFe 2 -AFs. All of these results therefore indicate that the fluorophore-labeled apo form of lactoferrin has an extra fluorophore (either AF350 or AF430) than found in fluorophore-labeled LfFe and $\mathrm{LfFe}_{2}$. The three lactoferrins labeled with both AF350 and AF430 exhibited FRET (Fig. 3). Excitation at $327 \mathrm{~nm}$ produced emission at 440 and 540 nm. The FRET pattern and efficiency were found to be characteristic of every fluorescent lactoferrin.

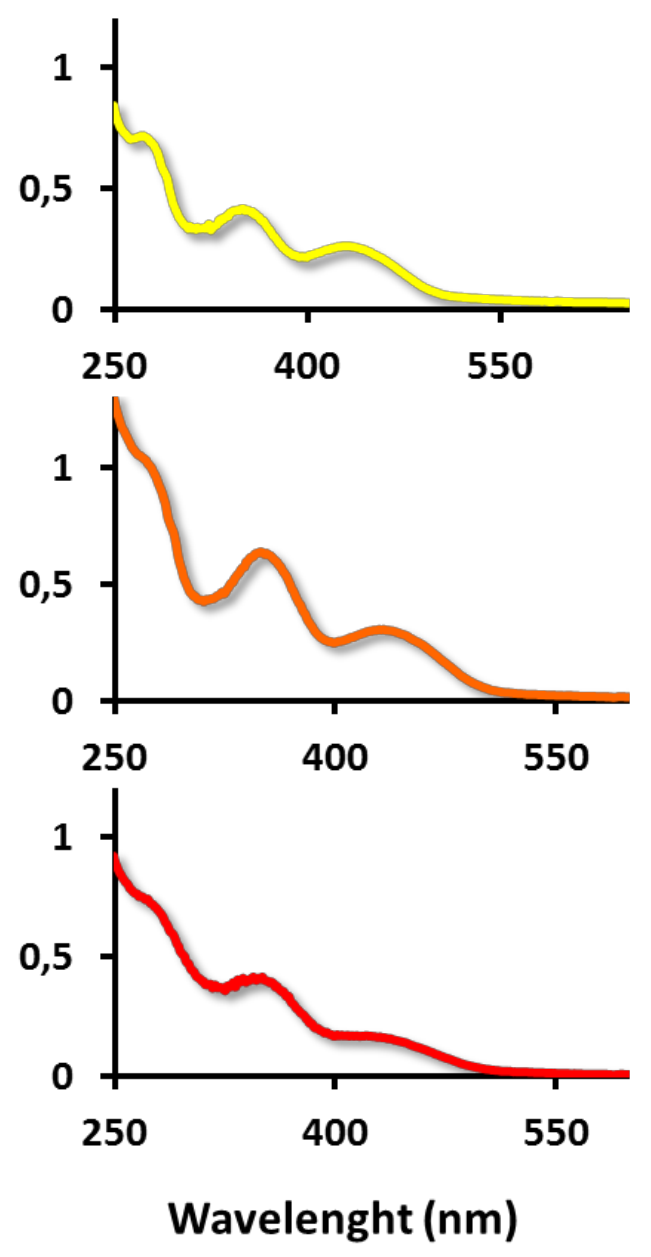

Fig. 2 UV-vis spectra of Lf-AFs indicating the number of AF350 and AF430 moities per protein in the combined AF350+AF430 labeling experiment. Concentrations of AF430, AF350, and protein were calculated from the absorbances at 430,350, and $280 \mathrm{~nm}$ (see "Materials and methods") 
In all cases, the emission at lower energy can be attributed to the AF430 acceptor and that at higher energy to the emission of the AF350 donor. In order to evaluate these different efficiencies, the \%FRET value of each lactoferrin was calculated using the following equation, which normalizes the intensity of the emission of AF430 ( $\left.\mathrm{I}_{\mathrm{AF} 430}\right)$ with respect to the intensity of the emission from both fluorophores $\left(\mathrm{I}_{\mathrm{AF} 350}+\mathrm{I}_{\mathrm{AF} 430}\right)$ :

$$
\% \mathrm{FRET}=\frac{I_{\mathrm{AF} 430}}{I_{\mathrm{AF} 350}+I_{\mathrm{AF} 430}} \times 100
$$
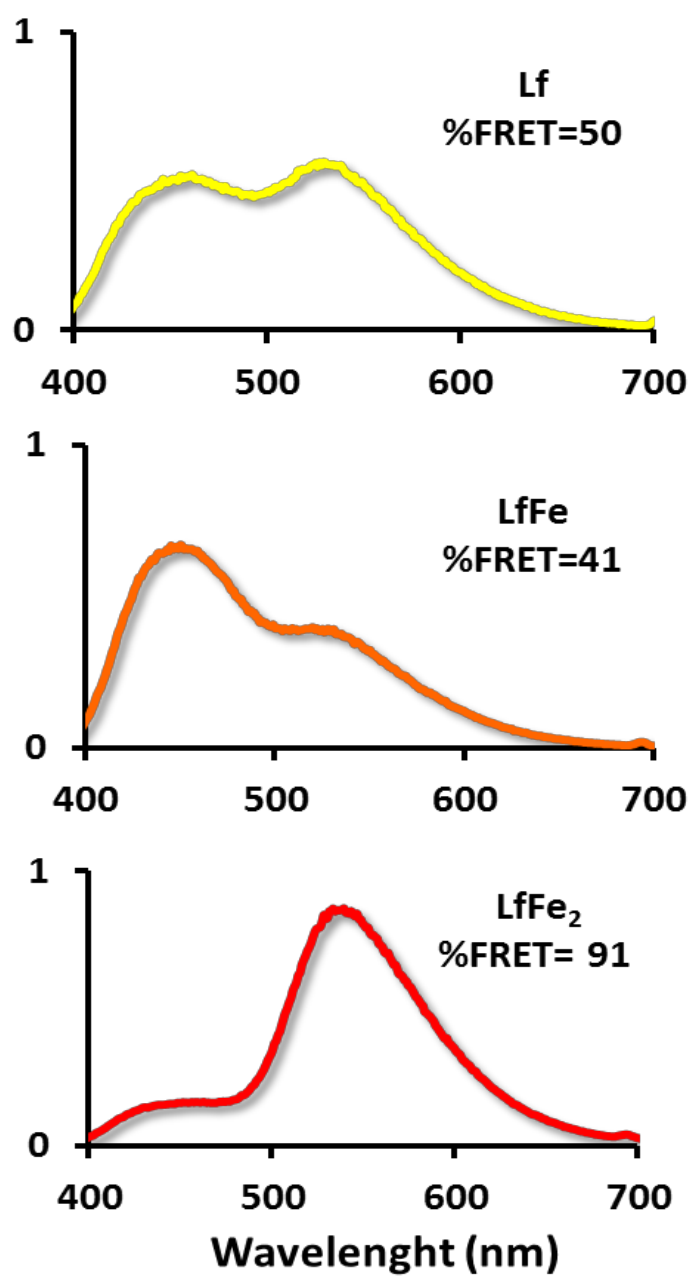

Fig. 3 FRET fluorescence emission spectra of the Lf-AF350+AF430, LfFeAF350 + AF430 and LfFe2-AF350 + AF430 samples

The calculated \%FRET yield values obtained from the data shown in Fig. 3 imply that the efficiency of the energy transfer between both fluorophores varies. Whereas complete energy transfer from the excited AF350 to AF430 does not 
occur in Lf-AFs (\%FRET $=50)$ or in LfFe-AFs (\%FRET $=41)$, practically complete energy transfer occurs in LfFe 2 -AFs $(\%$ FRET = 91). Consequently, the FRET pattern represents a "fingerprint" of the particular fluorescent lactoferrin that produces it, and is related to the conformational changes that occur to the protein upon the incorporation of iron.

Once these results had been analyzed, we aimed to correlate the energy transfer efficiency with the distance between the donor (AF350) and the acceptor (AF430), which obviously should be different in the three iron-loaded forms of Lf.

In order to gain greater insight into the differences observed in the number of fluorophores and the FRET patterns observed among the distinct iron-loaded states of lactoferrin, a computational analysis was performed. Calculations were first employed to ascertain the location of the binding site of the extra AF430 that is present in Lf-3AF350+3AF430 as compared to LfFe- and $\mathrm{LfFe}_{2}$ $3 A F 350+2 A F 430$. Second, computation was used to identify the region (or regions) of the protein that influences the FRET efficiency.

First, a comparative analysis of the solvent-accessible surface (SAS) for the 46 lysines in the X-ray structures of Lf and $\mathrm{LfFe}_{2}$ was performed (see SI1 in the Electronic supplementary material, ESM). As has been reported previously, succinimidyl ester derivatives of AFs are excellent dyes for labeling proteins through reactions with the available lysines [29]. To identify the location of the additional AF430 in the apo-Lf form, we hypothesized that the sixth lysine (i.e., the one to which the additional AF430 binds) should show a dramatic decrease in solvent accessibility when the apo form converts to the holo form of lactoferrin (in other words, upon changing from Lf-AFs to between the donor (AF350) and the acceptor (AF430), which obviously should be different in the three iron-loaded forms of Lf.

In order to gain greater insight into the differences observed in the number of fluorophores and the FRET patterns observed among the distinct iron-loaded states of lactoferrin, a computational analysis was performed. Calculations were first employed to ascertain the location of the binding site of the extra AF430 that is present in Lf-3AF350+3AF430 as compared to LfFe- and LfFe2-3AF350+2AF430. Second, computation was used to identify the region (or regions) of the protein 
that influences the FRET efficiency.

First, a comparative analysis of the solvent-accessible surface (SAS) for the 46 lysines in the X-ray structures of Lf and $\mathrm{LfFe}_{2}$ was performed (see SI1 in the Electronic supplementary material, ESM). As has been reported previously, succinimidyl ester derivatives of AFs are excellent dyes for labeling proteins through reactions with the available lysines [29].

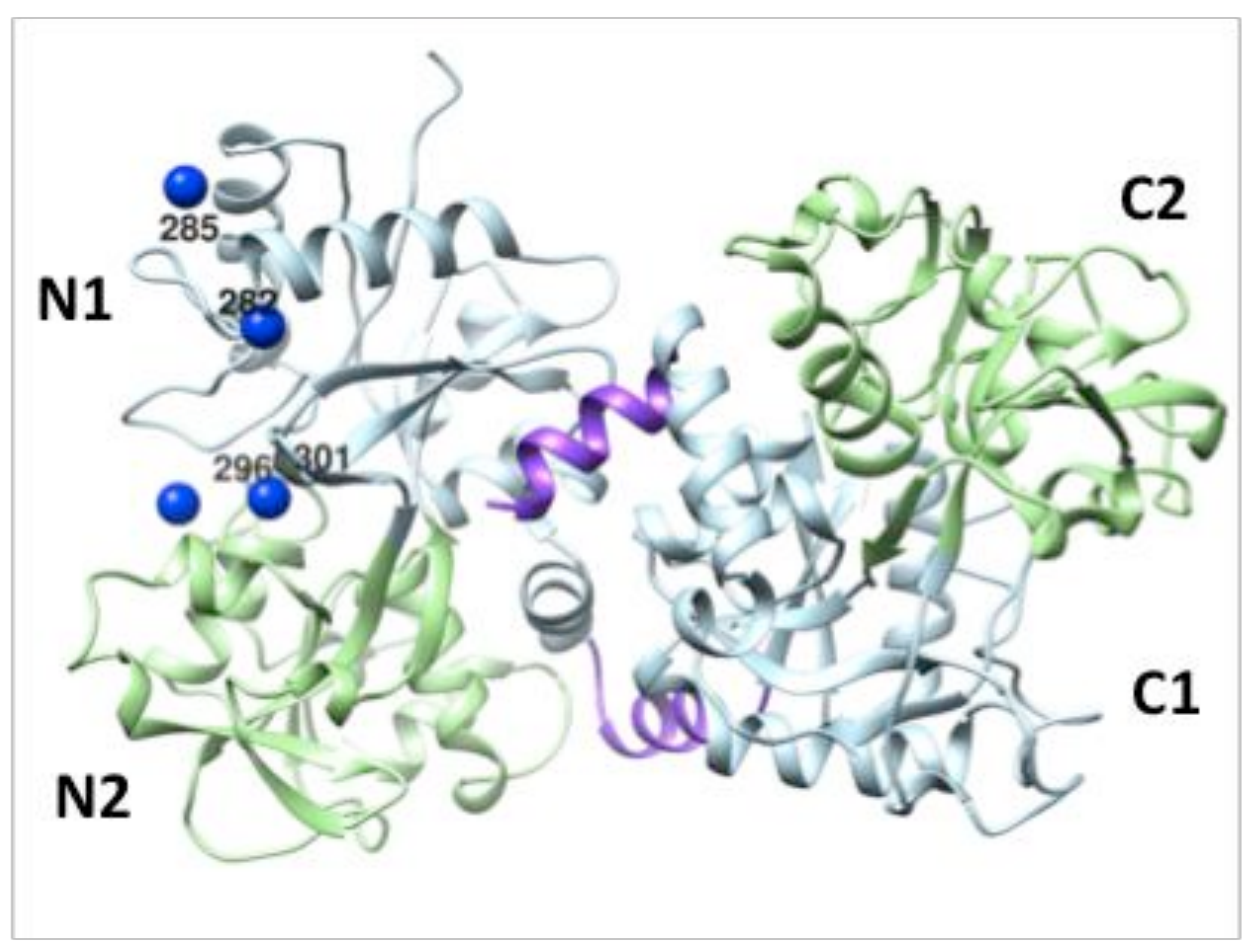

Fig. 4 Locations of the four lysines whose solvent accessible surface (SAS) values decrease the most upon converting from the $L f$ to the LfFe2 form. The backbones are represented by ribbons. Regions colored green correspond to the C2 and N2 subdomains, and those colored silver correspond to the C1 and N1 subdomains. The lysines of interest are represented as blue spheres at the Ne position and are labeled.

To identify the location of the additional AF430 in the apo-Lf form, we hypothesized that the sixth lysine (i.e., the one to which the additional AF430 binds) should show a dramatic decrease in solvent accessibility when the apo form converts to the holo form of lactoferrin (in other words, upon changing from LfAFs to $\mathrm{LFFe}_{2}$-AFs). A program written in Python in house and implemented in the UCSF Chimera environment [30] was designed for this analysis. Such an analysis 
could not be done of the monoferric species since no X-ray structure of LfFe was available.

Although most of the lysines were found to be highly exposed to the solvent, four residues (Lys282, Lys285, Lys296, and Lys301) presented a noticeable decrease in SAS during the conversion from Lf to $\mathrm{LFe}_{2}$. All of these residues are found in the $\mathrm{N}$-lobe, and more specifically in the $\mathrm{N} 1$ region of the protein: Lys282 and Lys285 are located at one of the external faces of the subdomain and Lys296 and Lys301 at the entrance and core of the iron-binding site, respectively (Fig. 4). Significantly, Lys282, Lys285, and Lys296 exhibit large SAS values, thereby suggesting that interaction with a fluorophore should be independent of the protein conformation. In contrast, Lys301 exhibited a large SAS value in Lf but a value of almost zero in $\mathrm{LFe}_{2}$, meaning that this residue changes from being highly exposed in the apo form (Lf) to deeply buried in the holo one ( $\left.\mathrm{LfFe}_{2}\right)$.

To better ascertain whether Lys301 is the Lys to which AF430 binds, and to gain a better understanding of its molecular features, protein-ligand dockings of the fluorophore at this site were performed using the available crystal structures of Lf and $\mathrm{LfFe}_{2}$. As stated in the "Materials and methods" section, these calculations were undertaken using the program GOLD and the Chemscore scoring functions [30]. A restraint was added between $\mathrm{N}$ of the lysine and the carbon atom of the carbonyl group of the fluorophore during the run in order to achieve geometries consistent with pre-reaction AF430 binding states. 


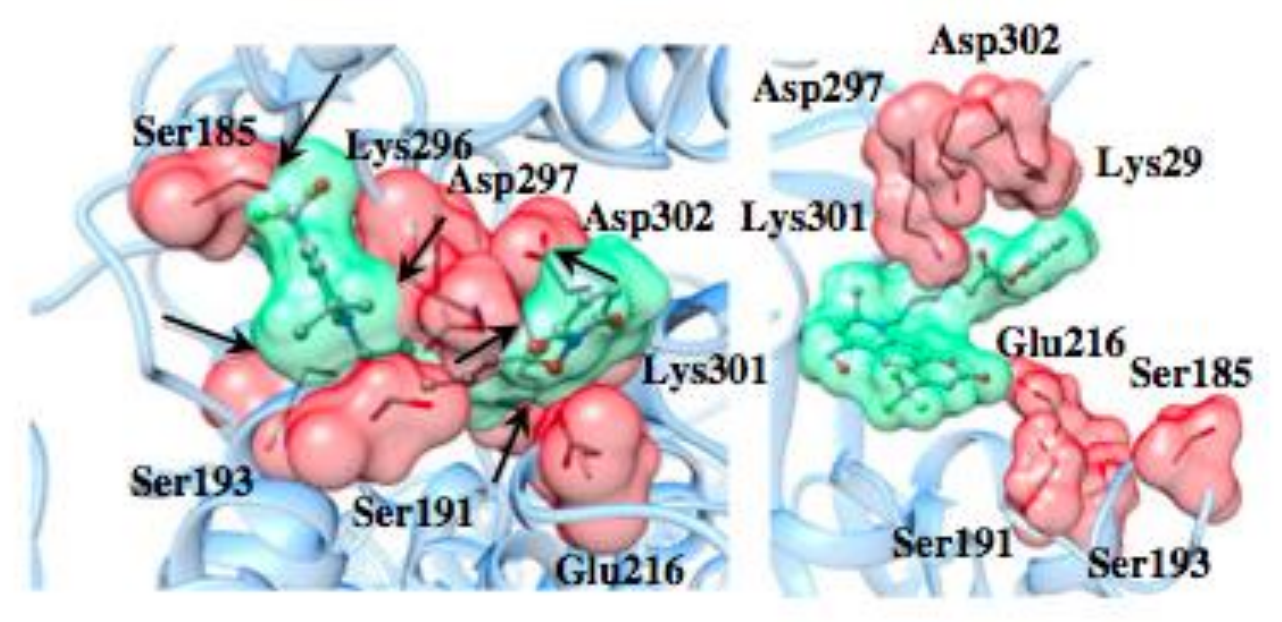

Fig. 5 Lowest-energy solutions for the conformation of AF430 that are consistent with its binding to Lys301 in LfFe2 (left panel) and Lf (right panel). The fluorophores are represented as balls and sticks and the protein residues as sticks. The surfaces are also depicted in order to highlight the residues (red) that show the most steric hindrance with the fluorophore (blue) in LfFe2

The predicted binding affinities for AF430 binding at Lys301 range from 18.8 to 22.1 scoring units on going from Lf to $\mathrm{LfFe}_{2}$, respectively. For calculations performed with the Chemscore function, these values imply a predicted binding affinity that could reach the micromolar range for the former but that binding is impossible for the latter [31].

These findings were further confirmed by repeating the calculations with AF430 on $\mathrm{LfFe}_{2}$, but adding greater flexibility to the Lys301 residue during docking (Fig. 5). Although the predicted affinity of the best binding mode increased at $13.75 \mathrm{U}$, the binding affinity was still low and was substantially different to that obtained for Lf. Moreover, for the conjugation to occur, Lys301 must escape from a buried situation in $\mathrm{LfFe}_{2}$ (where it interacts strongly with a network of polar interactions with the carboxylic residues E216 and D297) to a conformation that is highly exposed to the solvent. Marked steric hindrance is observed for the environments of the iron-binding sites in the resulting geometry. These results confirm that conjugation of the dye at this position is only possible in the apo form of Lf.

Additional calculations were performed to identify the region of the protein that 
could be responsible for the change in FRET efficiency upon shifting from the apo-Lf to the holo-LfFe ${ }_{2}$ form. These calculations, which considered the variations in the distances between all lysine residues during the transition from $\mathrm{Lf}$ to $\mathrm{LfFe}_{2}$, were performed using an additional program written in house in Python and implemented in the UCSF Chimera package [32]. The script provided a $46 \times 46$ matrix of the entire set of Lys-Lys distances. The comparative analysis of the X-ray structures of Lf and $\mathrm{LfFe}_{2}$ showed that most of the 46 Lys residues have interresidue distances that only rarely vary by $>3$ Å on average when the iron loading of the protein changes, thus remaining within the Fö rster distance range (Fig. 6). However, eight Lys residues, namely Lys99, 100, 113, 197, 200, 237, 241, and 243 , showed a different pattern, with variations of between 5 and $10 \AA$. Also, Lys180 showed variations that were slightly larger than $4 \AA$.

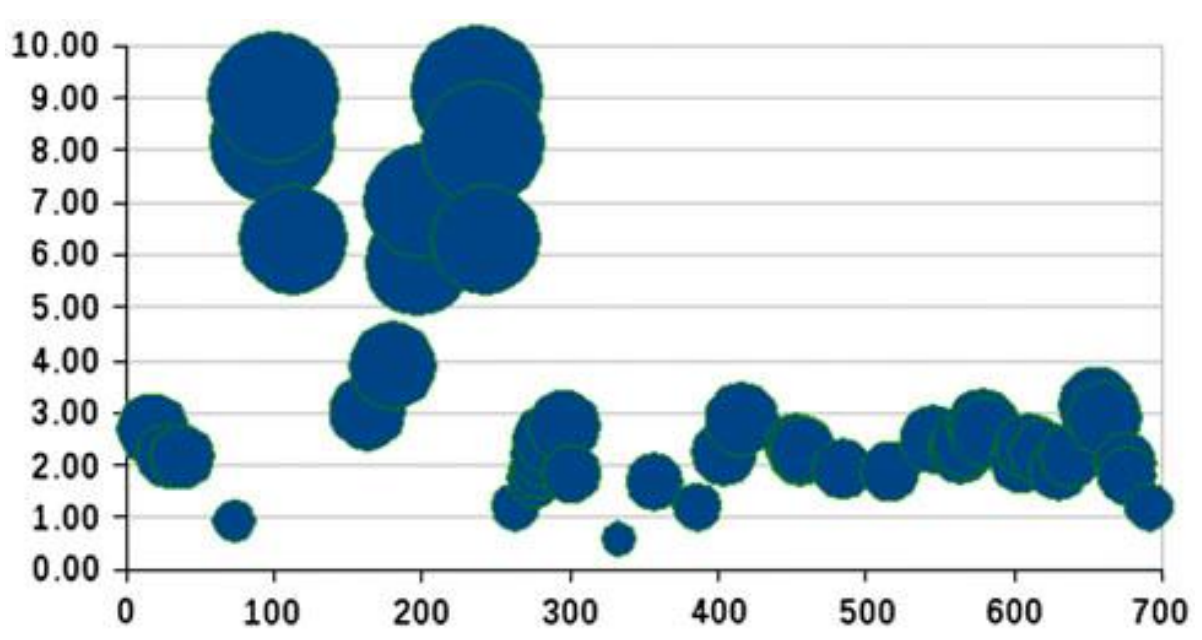

Fig. 6 Bubble chart showing the average variations in inter-residue distances upon transitioning from Lf to $\mathrm{LfFe}_{2}$. The size of each circle is proportional to the magnitude of the variation. Lys residue labels are given on the abscissa 


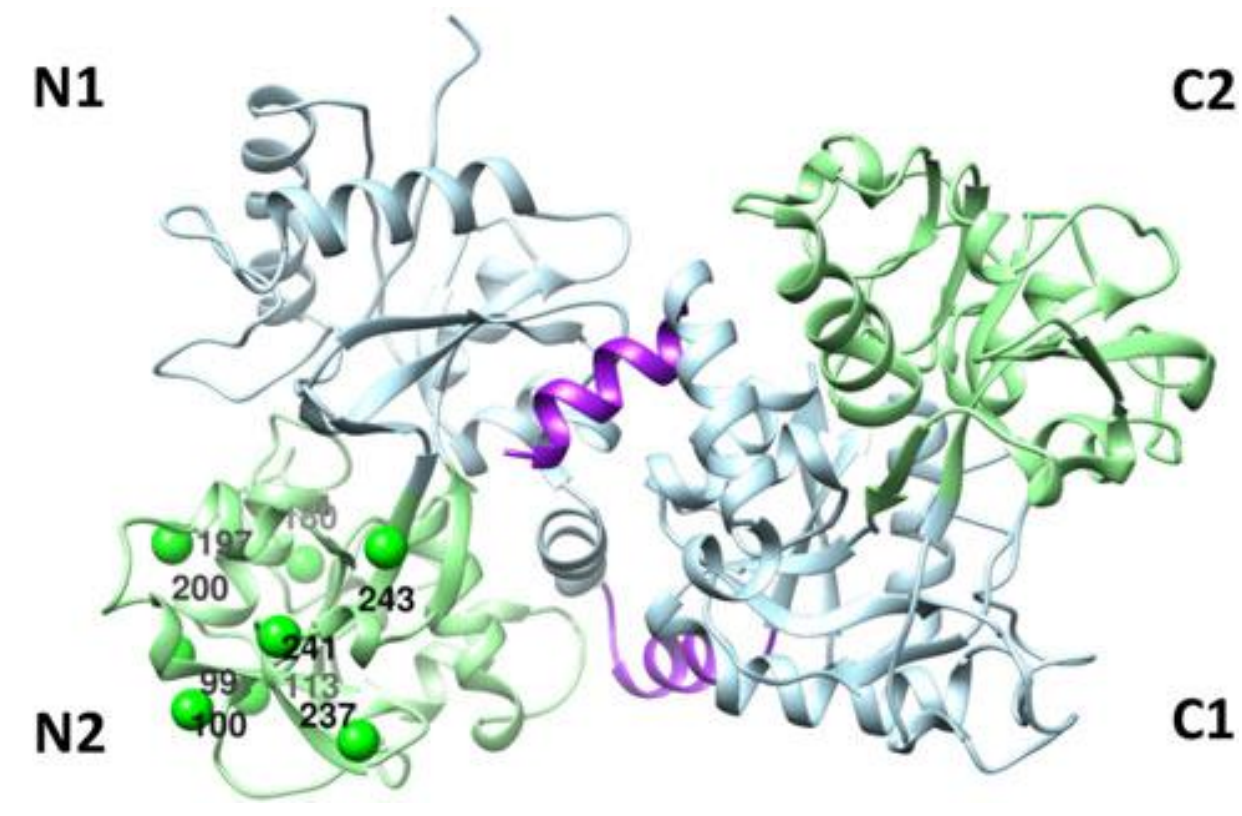

Fig. 7 Locations of the nine lysines whose average variations in inter-residue distance upon transitioning from Lf to LfFe 2 exceed $4 \AA$. The backbones are represented as ribbons. Regions colored green correspond to the C2 and N2 subdomains, and those colored silver correspond to the C1 and N1 subdomains. Putative lysines are represented as green spheres at the Ne position and are labeled

Importantly, these eight residues were all found to be located in the N2 subregion of the N-lobe. This subregion makes the greatest contribution to the conformational transition from Lf to $\mathrm{LfFe}_{2}$ (Fig. 7). Lys301, which was previously identified as the binding site for the "extra" AF430, is located at the interface between the N1 and N2 subdomains. Both of these results suggest that, even upon shifting to the LfFe form of the metalloprotein, a conformational change occurs to the macromolecule consisting of a subdomain rearrangement of the $\mathrm{N}$-lobe.

Using our improved understanding of the relationship between the FRET profile and iron loading, the possibility of using fluorophore labeling to monitor iron loading in lactoferrin-dependent processes was investigated. In particular, we monitored the process by which lactoferrin can supply iron to a healthy bacterium, in this case $B$. breve. The bacteria were incubated with $\mathrm{LfFe}_{2}-\mathrm{AFs}$ for different durations (1-6 h) and the FRET patterns of the supernatant solutions obtained after bacterial centrifugation were analyzed. After $6 \mathrm{~h}$, the resulting 
supernatant solution exhibited a FRET pattern typical of the iron-free Lf-AFs form (Fig. 8). A negligible amount of iron was detected in this solution (concentration below 10-9 M), while the concentration of the protein (Lf) remained constant. This finding suggests that all of the iron initially bound to lactoferrin is released and incorporated into the bacteria after this time. Likewise, no fluorescence was observed from the centrifuged bacteria. Interestingly, we observed that the FRET pattern does not change gradually but sharply, in two steps: from $\mathrm{LfFe}_{2}$ to LfFe, and then from LfFe to Lf (SI2).

\section{Discussion}

The present study of the FRET signals obtained when functionalizing lactoferrin with two distinct fluorophores, AF430 and AF350, provides not only novel molecular information on the iron-loading state of the protein but also on the mechanisms underlying these iron loading/unloading processes. Furthermore, the results illustrate how this methodology can be useful when performing in vivo and in vitro experiments involving Lf iron release or loading.

Our data show that both the change in the number of fluorophores that can be bound to lactoferrin and the variations in their FRET patterns as a function of the iron loading level of the protein are strongly dependent on the protein conformation. This means that the FRET responses can be used to analyze the iron-dependent conformational change of lactoferrin when it converts from its apo form to its holo (diferric) form, and can shed light on previously unanswered questions about this process. 

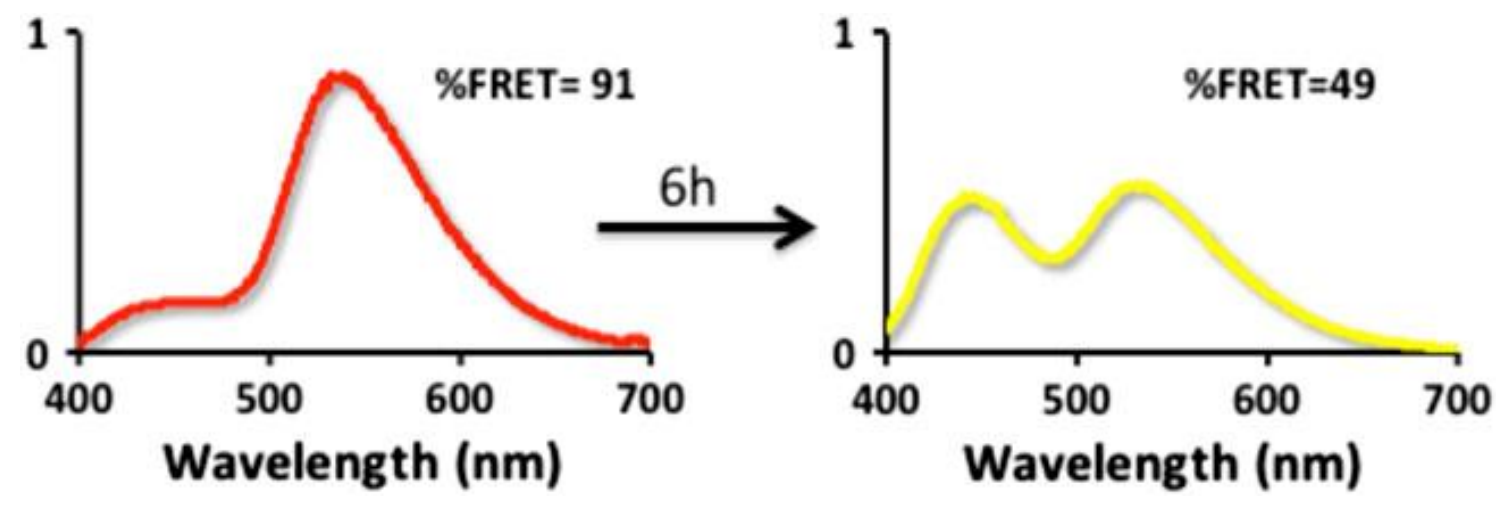

Fig. 8 FRET fluorescence emission spectra for LfFe2-AFs before (left) and after (right) incubation with B. breve for $6 \mathrm{~h}$

More precisely, the FRET responses can be used to probe how, following the uptake of the first iron(III) ion, the other lobe of the protein is prompted to bind the second ion. To the best of our knowledge, none of the information currently available provides a definitive answer to this question.

The results of our computational study, based on an SAS analysis of the Lys residues in $\mathrm{Lf}$ and $\mathrm{LfFe}_{2}$, clearly indicate that Lys301 is the only residue that is able to selectively bind AF430 in Lf (but not in $\mathrm{LFe}_{2}$ ). This result is supported by those obtained from simulated protein-ligand dockings that explicitly modeled the binding of the fluorophore to the selected amino acid and showed that AF430 can easily access a catalytically consistent orientation for binding at Lys301 in Lf but not in LfFe nor LfFe 2 (Fig. 5). Since Lys301 is located at the iron-binding site of the N-lobe and is located at the interface between the N1 and N2 subdomains, it appears that some type of conformational change occurs in this domain as a consequence of the binding of the first iron to the protein. Additional calculations on the available X-ray structures also allowed us to demonstrate that the differences in the FRET patterns obtained for $\mathrm{Lf}, \mathrm{LfFe}$, and $\mathrm{LfFe}_{2}$ are likely to be related to the fluorophores attached to the Lys residues located at the N2 lobe. This suggests that the transition between $\mathrm{Lf}$ and $\mathrm{LfFe}_{2}$ is likely to involve a major contribution from an interdomain change in the N-lobe.

At this point, it is interesting to note that uptake of the first iron(III) by lactoferrin 
has been reported to occur at the $\mathrm{C}$-site, at which point the $\mathrm{N}$-site becomes capable of acquiring a second iron(III) ion [33]. However, the mechanism by which the N-lobe is activated once this first iron(III) ion has been bound to the Clobe is not well known and the source of debate. To date, it is believed that the binding of the first iron(III) is followed by a series of proton dissociations that probably trigger changes in the conformation of the protein, thereby affecting the $\mathrm{N}$-site and allowing the capture of the second iron(III) [18].

On the one hand, our results suggest that the capture of the first iron(III) ion triggers a partial but significant enough interdomain change at the N-lobe. Lys301, which is located at the interface between the N1 and N2 subdomains, binds an AF430 in Lf but not in $\mathrm{LfFe}_{2}$. The steric hindrance of Lys301 is associated to a certain degree with the closing motion that takes place between the $\mathrm{N} 1$ and N2 subdomains upon the shift from the Lf to the LfFe form of the protein, as shown by further SAS analysis.

Therefore, our results show that the binding of the first metal to Lf involves a reorganization of the $\mathrm{N} 2$ domain with respect to the $\mathrm{N} 1$ one. However, the FRET efficiency remains almost unaffected (50 vs $41 \%$ ) by the Lf to LfFe transition, leading us to suspect that the conformational change is relatively limited.

The capture of the second iron(III) by the N-lobe results in a significant conformational change of the protein as a whole. A drastic increase in FRET is observed upon transitioning from $\mathrm{LfFe}(41 \%)$ to $\mathrm{LfFe}_{2}$ (91\%). This suggests that a marked change in the N2/N1 interface occurs with the binding of the second iron. In the absence of an X-ray structure for human LfFe, the results of our joint computational-experimental study suggest that the structure of LfFe presents an intermediate conformation of the $\mathrm{N}$-lobe that is mainly characterized by intersubdomain reorganization. This observation is consistent with the recently released structure of an intermediate conformation of di-iron and di-bismuth human transferrin, where the main structural changes were observed at the N2 domain and at the interface between both subdomains [34]. Our interpretations are also consistent with those of other authors, who have envisaged the possibility that cooperativity between the C- and N-lobes occurs via dynamic event modifications of the interlobe chain, rather than the lobes themselves $[18,33]$. 
In addition to the novel information obtained on the molecular mechanism of iron loading by lactoferrin, the present study illustrates how the FRET profile of lactoferrin can be used as a tool to monitor some specific processes of iron metabolism in which this protein plays a relevant role. In particular, lactoferrin may support the growth of some nonpathological but beneficial bacteria $[14,15]$. Indeed, lactoferrin coexists with several lactic acid bacteria, such as Bifidobacterium and Lactobacilli, in breast milk. These Gram-positive anaerobic bacteria proliferate in the human intestinal tract and are classified as probiotic bacteria because of their health benefits [35-37]. Our results confirm that, as for other healthy bacteria, human iron-saturated lactoferrin acts as an iron donor for B. breve. The evolution of the FRET pattern of the supernatant solution after the incubation of $\mathrm{LfFe}_{2}$ with the bacteria (Fig. 8) points to complete iron transfer from $\mathrm{LfFe}_{2}$ to the bacteria. Moreover, the fact that the protein concentration in the extrabacterial medium does not significantly change suggests that lactoferrin does not require protein internalization and that, unlike other transferrins, it is not involved in Fe(III) transport by receptor-mediated endocytosis. This kind of iron trafficking has only been observed in certain Gram-negative bacteria [38].

\section{Conclusions}

Fluorescent lactoferrins with different iron loading levels were prepared by labeling the proteins with the Alexa Fluor dyes AF350 and AF430. All of the fluorescent lactoferrin samples showed FRET, but interestingly with distinctive patterns. This indicates that energy transfer from the donor to the acceptor fluorophore varies with the iron loading level of lactoferrin. Since the iron loading level of lactoferrin determines its tertiary structure, the FRET pattern of the sample correlates with its iron content. Moreover, the results of our joint experimental-computational study provide an additional piece of evidence relating to the subdomain dynamics of the N-lobe upon iron binding. They are consistent with a cooperative transfer mechanism between the $\mathrm{C}$ - and N-lobes of lactoferrin that involves interdomain conformational changes during the transition from the Lf to the LfFe form, although the closed conformation found in $\mathrm{LfFe}_{2}$ is not achieved during this transition. 
In a further achievement, we used this FRET to iron level correlation for lactoferrin as a tool to monitor the interaction between human lactoferrin and Bifidobacteria. Specifically, we used this methodology to demonstrate that lactoferrin supplies iron to these healthy bacteria and, furthermore, that this process takes place without iron(III) trafficking by receptor mediated endocytosis.

\section{Acknowledgments}

This work was supported by MINECO and FEDER (projects CTQ2012-32236, CTQ2011-23336, and BIO2012- 39682-C02-02) and BIOSEARCH SA. F.C. and V.M.R. are grateful to the Spanish MINECO for FPI fellowships

\section{References}

1. Vogel H (2012) J Biochem Cell Biol 90:233-244

2. Baker EN, Baker HM (2005) Cell Mol Life Sci 62:2531-2539

3. Baker EN, Baker HM (2009) Biochimie 91:3-10

4. Anderson BF, Baker HM, Norris GE, Rice DW, Baker EN (1989) J Mol Biol 209:711-734

5. Baker EN, Lindley PF (1992) J Inorg Biochem 47:147-160

6. Birgens HS (1985) Scand J Haematol 34:326-331

7. Orsi N (2004) Biometals 17:189-196

8. Byrd TF, Horwitz MA (1991) J Clin Investig 88:351-357

9. Griffiths E, Duffy L, Schanbacher F, Dryja D, Leavens A, Neiswander R, Qiao H, DiRienzo D, Ogra P (2003) Dig Dis Sci 48:1324-1332

10. Arnold RR, Cole MF (1977) Science 197:263-265

11. Valenti P, Antonini G (2005) Cell Mol Life Sci 62:2576-2587

12. Berkhout B, Floris R, Recio I, Visser S (2004) Biometals 17:291-294

13. Kanyshkova TG, Semenov DV, Buneva VN, Nevinsky GA (1999) FEBS Lett 451:235-237 
14. Liepke C, Adermann K, Raida M, Mägert H-J, Forssmann W-G, Zucht H-D (2002) Eur J Biochem 269:712-718

15. Sherman M, Bennett S, Hwang FY, Yu C (2004) Biometals 17:285-289

16. Kumar J, Weber W, Mü nchau S, Yadav S, Singh SB, Saravanan K, Paramasivam M, Sharma S, Kaur P, Bhushan A, Srinivasan A, Betzel C, Singh TP (2003) Indian J Biochem Biophys 40:14-21

17. Chug TDY, Raymond KH (1993) J Am Chem Soc 115:6765-6768

18. Bou AF, El Hage Chahine JM (2000) J Mol Biol 303:255-266

19. Moosmann A, Blath J, Lindner R, Mü ller E, Bö ttinger H (2011) Bioconjug Chem. 22:1545-1558

20. Breuer W, Cabantchik ZI (2001) Anal Biochem 15:194-202

21. Nojima Y, Suzuki Y, Iguchi K, Shiga T, Iwata A, Fujimoto T, Yoshida K, Shimizu H, Takeuchi T, Sato A (2008) Bioconjug Chem 19:2253-2259

22. Zalipsky S (1995) Bioconjug Chem 6:150-165

23. Xavier PL, Chaudhari K, Verma PK, Pal SK, Pradeep T (2010) Nanoscale 2:2769-2776

24. Myc A, Majoros IJ, Thomas TP, Baker JR Jr (2007) Biomacromolecules 8:13-18

25. Wang YA, Li JJ, Chen H, Peng X (2002) J Am Chem Soc 124:2293-2298

26. Green M, Howman E (2005) Chem Commun 121-123

27. Bates GW, Billups C, Saltman P (1967) J Biol Chem 242:2816-2821

28. Faller B, Nick H (1994) J Am Chem Soc 116:3860-3865

29. Fernández B, Gálvez N, Sánchez P, Cuesta R, Bermejo R, Domínguez-Vera JM (2008) J Biol Inorg Chem 13:349-355

30. Pettersen EF, Goddard TD, Huang CC, Couch GS, Greenblatt DM, Meng EC, Ferrin TE (2004) J Comput Chem 25:1605-1612

31. Maréchal JD, Yu J, Brown S, Kapelioukh I, Rankin E, Wolf C, Roberts G, Paine M, Sutcliffe M (2006) Drug Metab Dispos 34:534-538

32. Verdonk ML, Cole JC, Hartshorn MJ, Murray CW, Taylor RD (2003) Proteins 
$52: 609-623$

33. Pakdaman R, Petitjean M, El Hage Chahine JM (1998) Eur J Biochem 254:144 153

34. Yang N, Zhang H, Wang M, Hao Q, Suna H (2012) Sci Rep 2:999

35. Macfarlane S, Macfarlane GT, Cummings JH (2006) Aliment Pharmacol Ther 24:701-714

36. Shah NP (2000) J Dairy Sci 83:894-907

37. Kaur IP, Chopra K, Saini A (2002) Eur J Pharm Sci 15:1-9

38. Heymann JJ, Weaver KD, Mietzner TA, Crumbliss AL (2007) J Am Chem Soc 129:9704-9712 